\title{
Synthesis of Photoactive SPIONs Doped with Visible Light Activated Photosensitizer
}

\section{Kumar P*, Agnihotri S and Roy I}

Department of Chemistry, University of Delhi, India

\begin{abstract}
We have synthesized low cost and water dispersed citric acid coated iron-oxide nanoparticles at room temperature. TEM data were 10 shown the average diameter of these nanoparticles around $15 \mathrm{~nm}$ and XRD were then used to the crystalline nature of these particles. VSM experiment showed that these nanoparticles are superparamagnetic, with high magnetization $(78 \mathrm{emu} / \mathrm{g})$. In vitro studies have shown that these nanoparticles are nontoxic to human cells in culture. The citric acid coated superparamagnetic iron-oxide nanoparticles (CA-SPIONs) conjugated with a photosensitizer (PS-CA-SPIONs) for applications in photodynamic therapy (PDT). These PS-CA- SPIONs, stably-dispersed in aqueous medium, was characterized using optical studies. An Experiment involving light-activated 15 generation of reactive oxygen species (ROS) revealed that only the PS-CA-SPIONs, but not free PS, could generate the cytotoxic singlet oxygen. Cell viability studies demonstrated PDT-mediated cytotoxic effects on cells treated with PS-CA-SPIONs and irradiated with light. The results indicate the potential of PS-CA-SPIONs as delivery agents in (photodynamic therapy.
\end{abstract}

Keywords: SPIONs; PS-CA-SPIONs; Photodynamic therapy

\section{Introduction}

Nanomaterials are basically those nanoparticles whose properties are evident by the nano objects they have. Among the nanomaterials reported so for the metal oxide nanoparticles have shown great significance because of potential applications of these nanoparticles in various applications [1]. A lot of research studies have been focused on the use of a variety of metal oxide nanoparticles but recently the use of Magnetic metal oxide has shown great attraction as these magnetic metal oxides with modified surface as well as core. These nanoparticles are the potential next generation anticancer agents [2,3] along with their use in various other diagnostic assays such as MRI contrast agents [4], hyperthermia, [5] cell differentiation [6] and targeted drug delivery [7]. Agglomeration of nanoparticles is the major demerit of these nanoparticles as it results in loss of magnetic efficiency, activity and rapid elimination. Thus to overcome these challenges and to maintain the superparamagnetic behavior along with high stability of magnetic metal oxide nanoparticles we have taken iron oxide nanoparticles as these particles avoid ferromagnetic agglomeration and due to being sterically 30 stable these particle don't agglomerate in physiological media [8] In environment iron oxide basically exit in iron (II) and iron (III) oxidation state [9]. As earlier reported in so many studies that iron oxide nanparticles are also refer as super paramagnetic iron oxide nanoparticles i.e. SPIONs. SPIONs are the most significant metal oxides as they are used in lots of technological and biomedical applications. Thus the major advantages of taking iron oxide nanoparticles (IO NPs) are that these SPIONs are highly stable, low cost wide spread material, biodegradable and relatively low toxic to the human cells [10]. Thus these SPIONs were commonly synthesized by the co-precipitation method, using ferrous and ferric salts to attain the smaller size nanoparticles. These particles are super paramagnetic in nature and the force acting on nanoparticle is directly proportional to particle size in a particular applied magnetic field, thus these nanoparticles requires large applied magnetic field for in vivo studies. Thus the large volume of SPIONs is ferromagnetic in nature and to overcome the agglomeration the SPIONs were coated with citric acid which acts as stabilizer [1113]. Therefore, magnetic metal oxide nanoparticles such as SPIONs are widely used in so many biomedical applications [14].

Photodynamic therapy is a type of light activated therapy in which a light absorbing moiety that is a photosensitizer (PS) is used which absorbs the light of a particular wavelength usually in visible region or near infrared (NIR) region and get excited [15]. The excited PS then transferred its excited-state energy to the neighboring oxygen atom which triggers the oxidative photo damage by the formation of a very reactive form of oxygen i.e singlet oxygen (1O2) or reactive oxygen species (ROS) responsible for the cytotoxic action in PDT results tumorous and cancerous cell death along with vascular damage [1620]. This approach is a minimally invasive therapeutic approach with very less side effects as it is highly localized and induces no significant toxicity to the healthy mammalian cells as compared to radio and chemo therapy for the treatment of cancerous cells. Although, this technique is not very effective for the treatment of solid tumor having thickness more than a few millimeters. Thus, in photodynamic therapy (PDT) both wavelengths of light from visible region and NIR region are used because visible region light cannot penetrate deeper inside and are effective for basically comparatively superficial tumors in the body therefore more penetrating NIR region light was used to get more penetration [21]. Thus, PDT is used as an alternative modality to effectively treat the superficial and localized tumors rather than the other classical techniques such as surgery, radio and chemo therapy. Firstly, it was used for the treatment for cancerous tissues but nowadays it is used in lots of other related clinical applications [22-28].

The photosensitizers are sparingly soluble in water like other chemotherapeutic agents present in the market and require an adequate pharmaceutical formulation to improve its pharmacokinetics while parenteral administration [29-31]. A few drawbacks are also 5 associated with photosensitizers that some PSs have a strong affinity for a particular tissue and get accumulated there results in photo

*Corresponding author: Kumar P, Department of Chemistry, University of Delhi, Delhi, India, Tel: 9711065966; E-mail: pramodgang03@gmail.com

Received May 27, 2016; Accepted July 04, 2016; Published July 12, 2016

Citation: Kumar P, Agnihotri S, Roy I (2016) Synthesis of Photoactive SPIONs Doped with Visible Light Activated Photosensitizer. J Nanomed Nanotechnol 7: 392. doi: 10.4172/2157-7439.1000392

Copyright: (C) 2016 Kumar $P$, et al. This is an open-access article distributed under the terms of the Creative Commons Attribution License, which permits unrestricted use, distribution, and reproduction in any medium, provided the original author and source are credited. 
Citation: Kumar P, Agnihotri S, Roy I (2016) Synthesis of Photoactive SPIONs Doped with Visible Light Activated Photosensitizer. J Nanomed Nanotechnol 7: 392. doi: 10.4172/2157-7439.1000392

damage of adjacent healthy tissues. To overcome these problems a novel approach is still needed for an efficient drug delivery in PDT. An efficient technique can be an externally guided drug delivery, which could be achieved by introducing magnetic properties or moieties to the nanocarriers followed by manipulating the pharmacokinetics by an externally applied magnetic field. This can be a very effective technique for magnetically accessible tumors which coincidently are targets for PDT. Therefore, developing such a system in 10 which a photosensitizer was encapsulated in a magnetic nanoparticle and externally controlling its concentration at target tissue along with light irradiation results in generation of singlet oxygen would be an efficient therapeutic technique.

\section{Experimental}

\section{Materials/Chemicals details}

Ferric chlorides $\left(\mathrm{FeCl}_{3} \cdot 6 \mathrm{H}_{2} \mathrm{O}\right)$, ferrous sulphate $\left(\mathrm{FeSO}_{4} \cdot 6 \mathrm{H}_{2} \mathrm{O}\right)$, ammonia solution (25\%), were all purchased from Alfa Aesar. Lung carcinoma cells lines (A 549) were purchased from ATCC, VA and remaining other cell culture products was purchased from Thermo Fisher scientific. Ammonium acetate $\left(\mathrm{CH}_{3} \mathrm{COONH}_{4}\right)$, DMSO (dimethyl sulphoxide) and concentrated hydrochloric acid $(\mathrm{HCl})$ were purchased from Merck. Fetal bovine serum (FBS), Dulbecco's modified eagle's medium (DMEM), phosphate buffer saline (PBS), 3-20 (4, 5-dimethylthiazol-2-yl), 5-diphenyltetrazolium bromide (MTT reagent), amphotericin- $\mathrm{B}$, penicillin and streptomycin were purchased from Sigma-Aldrich. Citric acid monohydrate (CA) was purchased from Spectrochem pvt. Ltd. Mumbai, India.

\section{Material synthesis/Reactions}

\section{Synthesis of citric acid coated SPIONs (CA-SPIONs)}

The chemical precipitation method was used for the synthesis of citric acid stabilized super paramagnetic iron oxide nanoparticles (CASPIONs). $2 \mathrm{mmol}$ of an aqueous solution of ferric chloride , $1 \mathrm{mmol}$ of $\mathrm{FeSO}_{4}$ was mixed along with $1 \mathrm{~mL}$ of $\mathrm{HCl}$ solution $(50 \mu \mathrm{L} 12 \mathrm{~N}$ $\mathrm{HCl}$ diluted to $1 \mathrm{~mL}$ by DDW) and $40 \mathrm{~mL}$ of double distilled water (DDW) within nitrogen atmosphere. Reduction of iron salt solution was carried out by the addition of $5 \mathrm{~mL}$ ammonia solution drop by drop with continuous vigorous stirring for a few minutes which results in a dark suspension of SPIONs. After it $1 \mathrm{~mL}$ aqueous $(0.5 \mathrm{mg} / \mathrm{mL})$ citric acid as a stabilizer, was added and kept on stirring for $30 \mathrm{~min}$. at room temperature. The solution thus obtained was centriguged at $8000 \mathrm{rpm}$ for $10 \mathrm{~min}$. and thoughly washed with ethanol (3 time) and finally resuspended in double distilled water [31].

\section{Synthesis of PS conjugated CA-SPION (PS-CA-SPION)}

The synthesis of PS-CA-SPIONs has been carried out by adding $1 \mathrm{~mL}$ of PS solution ( $2 \mathrm{mg} / \mathrm{mL}$ in DMSO) in aqueous dispersion of CA-SPIONs $(50 \mu \mathrm{L}$ of $2 \mathrm{mg} / \mathrm{mL})$ and kept at room temperature with continuous stirring for $20 \mathrm{~min}$. After centrifugation at $8000 \mathrm{rpm}$ for $10 \mathrm{~min}$. the suspension was thoroughly washed with 2-3 times with ethanol and then finally resuspended in water [29].

\section{Characterizations}

The magnetic properties of the dried nanoparticles were probed using vibrating sample magnetometer (VSM) and High resolution powder X-ray diffraction (XRD) was used to analyze the phase composition of the nanoparticles, using an X-ray spectrometer. TEM micrograph, composition of elements (using energy dispersive spectroscopy, or EDX) and crystalline diffraction pattern (SAED) of 45 nanoparticles were also taken to analyze the size of the nanoparticles on forrmvar coated $\mathrm{Cu}$ grids (for TEM) by using TECNAI G2-30 U TWIN TEM instrument (FEI, Eindhoven, The Netherlands) with an acceleration voltage of $300 \mathrm{kV}$. The optical properties were recorded using a spectrophotometer and Cary Eclipse fluorescence spectrometer respectively. The particle size and surface charge were analyzed by dynamic light scattering technique using NANO-ZS series MALVERN ZETASIZER instrument. A Brukar D8 Discover x-ray spectrometer was used to analyze the phase composition of nanoparticles over the $2 \theta$ range from $20^{\circ}-65^{\circ}$ at a rate of $2.58 / \mathrm{min}$, using $\mathrm{Cu}-\mathrm{Ka}$ radiation $(\lambda=1.54060 \AA)$. The optical properties of nanoparticles i.e. both UVvisible absorption and spectra were recorded using a Shimadzu UV1601 spectrophotometer and Cary Eclipse fluorescence spectrometer respectively. Helium-Ne 5laser was used as the light source of wavelength $635 \mathrm{~nm}$ and power $4 \mathrm{~mW}$.

\section{Result and Discussion}

The TEM micrograph clearly indicates that the morphology of the majority of particles is spherical and the average particle size of the synthesized iron-oxide nanoparticles is approximate $15 \mathrm{~nm}$ and the uniform distribution along with least aggregation (Figure 1) of the particles were inferred their well dispersive nature in an aqueous medium.

The magnetization curve obtained for CA-SPIONs was similar to the pattern of superparamagnetic materials as reported earlier. On applying large external field the magnetization of nanoparticles aligns with the direction of the applied field and reaches its saturation value of these nanoparticles. Thus the pattern corresponding to CA-SPIONs is an evidence of their super paramagnetic nature as shown in Figure 2 [30]. The saturation magnetization (Ms) values recorded for CASPIONs was $78.0 \mathrm{emu} / \mathrm{g}$.

The XRD spectrum of the CA-SPIONs which is showing diffraction peaks of $200,311,400,422,500$ and 440 corresponds to the 15 characteristic peaks (Figure 3) of inverse spinel oxide and $\mathrm{Fe}_{3} \mathrm{O}_{4}$. The diffraction peaks delineate cubic unit cells and face centered cubic (fcc) unit cells of nanoparticles. Thus, from the XRD pattern, it was evident that nanoparticles were crystalline in nature.

The UV-visible spectra of PS were taken in DDW, which showed

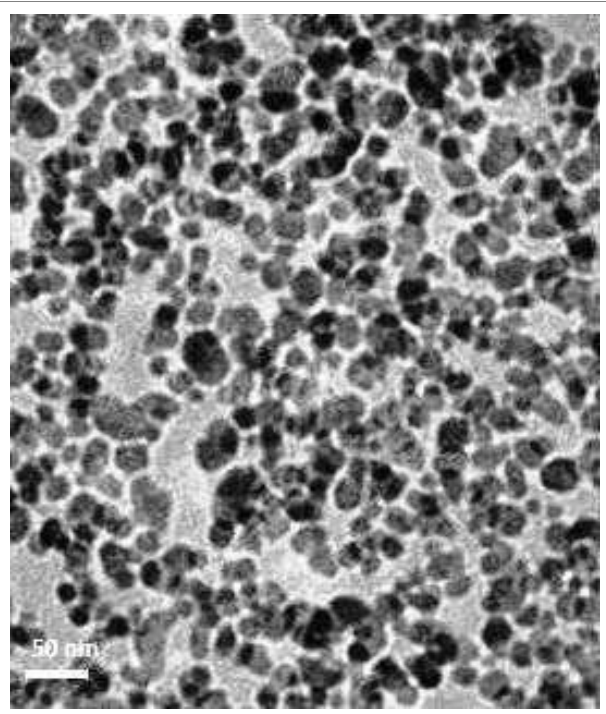

Figure 1: TEM images of CA-SPIONs showing average at scale $50 \mathrm{~nm}$. 
Citation: Kumar P, Agnihotri S, Roy I (2016) Synthesis of Photoactive SPIONs Doped with Visible Light Activated Photosensitizer. J Nanomed Nanotechnol 7: 392. doi: 10.4172/2157-7439.1000392

Page 3 of 4

characteristic absorption peaks at 438 and other bands 525, 567, 605, $669 \mathrm{~nm}$. As shown in the Figure 4 the presence of the corresponding peaks in lower intensity clearly indicates the conjugation of PS with the CA-SPION.

In Photodynamic therapy, a molecule absorbs photon and this photo exited molecule, i.e. photosensitizer (PS) transfer its excess energy to molecular oxygen via non-radioactive decay, leading to the generation of highly reactive oxygen species (ROS) that is singlet oxygen which can efficiently kill cancerous cells on targeted exposure to cancerous sites.

In this study, the investigation of singlet oxygen generated by PSCA-SPIONs was studied by the singlet oxygen mediated bleaching of the dye ABMDMA via measuring the time dependent decrease of absorption at $400 \mathrm{~nm}$ ( $\lambda$ max of ABMDMA). The chemical probe 25 9,10-anthracenediyl-bis (methylene) dimalonic acid (ABMDMA) which is a water soluble derivative of anthracene was reduced to its corresponding endo-peroxide by photo-bleaching done by singlet oxygen is work as indicator. The results have been shown in Figure 5 and it was evident that there is no significant change in absorbance for control set (ABMDMA with laser irradiation) rather than a marginal decrease in the absorbance was observed for the experimental set (PSCA-SPIONs-ABMDMA with laser irradiation). Thus, it was observed that bleaching of ABMDMA has been caused by the generated singlet oxygen from the IO-PS mixture rather than 30 irradiation only. Though this result demonstrates that there is no significant bleaching of ABMDMA alone, but it indicates the promising IO-mediated delivery

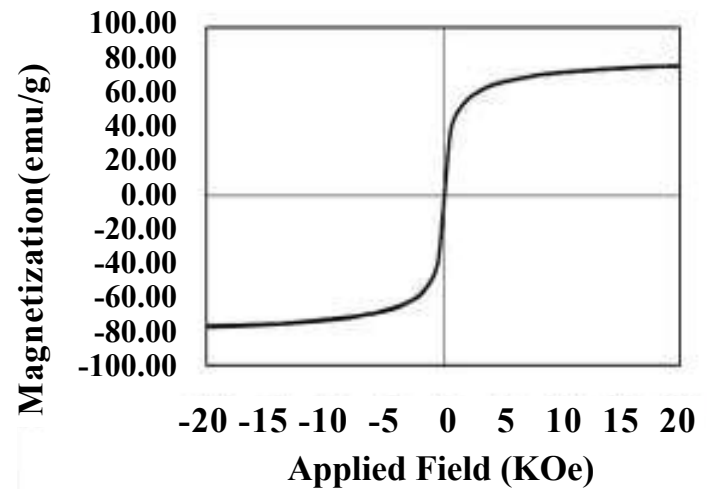

Figure 2: VSM Pattern of CA-SPIONs.

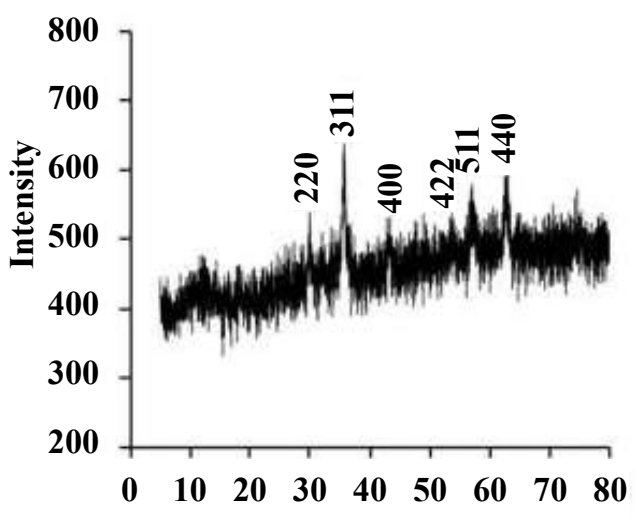

$2 \theta$

Figure 3: Power X-ray diffraction (XRD) of CA-SPIONs. of PS for PDT [31]. Thus, from the above observation, it can be inferred that the PS-CA-SPION have great potential for being a Photodynamic therapeutic agent for the treatment of cancer.

\section{In vitro toxicity}

The cells were seeded in 24-well plates at a confluence of about $30 \%$, the following samples were added per well in triplicates: (a) free PS $(0.15 \mu \mathrm{M})$, (b) CA-SPIONs NPs $(20 \mu \mathrm{g} / \mathrm{ml})$, and (c) PS-CA-SPIONs $(0.15 \mu \mathrm{M}$ PS conjugated on $20 \mu \mathrm{g} / \mathrm{ml}$ of $35 \mathrm{IO} \mathrm{NPs})$ and incubate for 14 hours and then irradiated with $635 \mathrm{~nm}$ laser light $(10 \mathrm{mins}$ in each well) using diode laser with output power of $20 \mathrm{~mW}$ followed by overnight incubation. Lung carcinoma cell lines (A549) were used for cell viability study and MTT reagent as the growth indicator. Figure 6 clearly indicates that the cells are more than $98 \%$ viable even at a high dosage of $10 \mu \mathrm{g} / \mathrm{mL}$.

This data demonstrates that the CA-SPIONs, PS, and PS-CASPIONs impose negligible toxic effect on the cells.

\section{Conclusion}

In this article, we describe the synthesis, characterization and in vitro applications of PS-CA-SPIONs. Further XRD and VSM data prove the crystalline and superparamagnetic behavior of PS-CA-SPIONs respectively. In vitro studies clearly prove that these nanoparticles are non-toxic to mammalian cell lines (A549). These PS-CA-SPION NPs on exposure with light generate highly reactive and lethal singlet

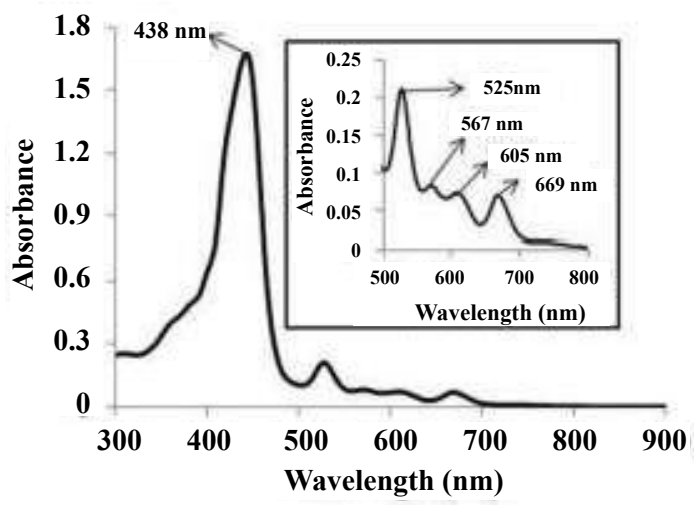

Figure 4: Absorbance spectra of PS in water solution.

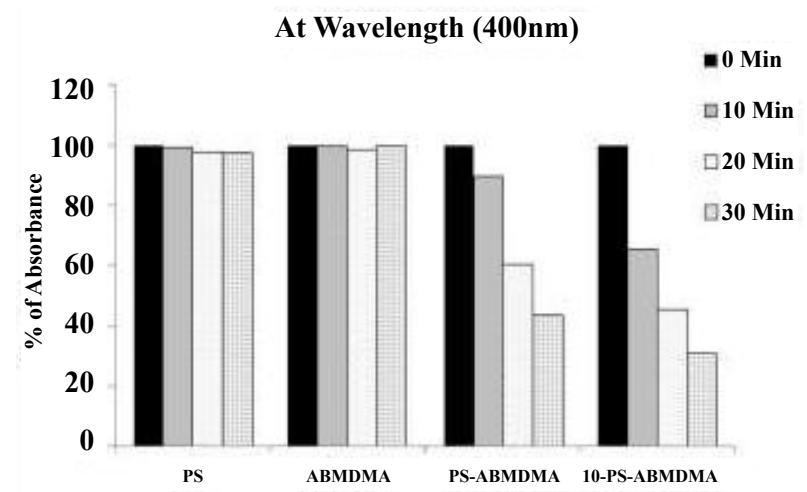

Figure 5: Combined results for all the three systems, showing decrease of $\mathrm{OD}$ at $400 \mathrm{~nm}$ (absorption maxima of 5 ABMDMA) as a function of laser irradiation time and Percentage of cell survival of A-549 cells, after treatment with Placebo nanoparticles (IONPS), free PS (PS) 
Citation: Kumar P, Agnihotri S, Roy I (2016) Synthesis of Photoactive SPIONs Doped with Visible Light Activated Photosensitizer. J Nanomed Nanotechnol 7: 392. doi: 10.4172/2157-7439.1000392

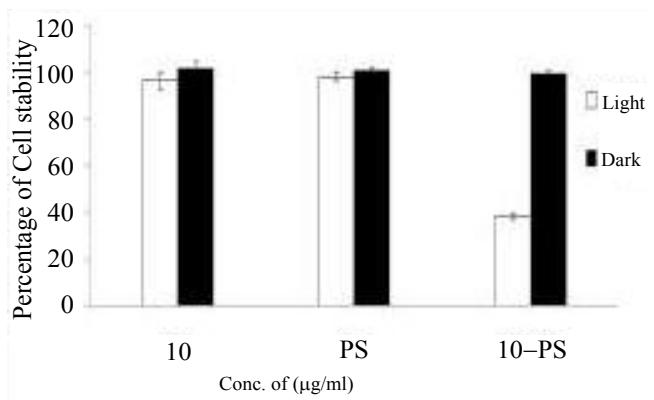

Figure 6: PS-Conjugated IO nanoparticles (PS-CA-SPIONs), with (light) and without (dark) subsequent irradiation with $630 \mathrm{~nm}$ laser light.

oxygen species which make them a potential photodynamic therapeutic agent and can be used in other biological applications.

\section{Acknowledgements}

We are grateful to the University of Delhi, India, for providing Research and Development grant RC/2015/9677 for support this study.

\section{References}

1. Kharisov BI, Dias HR, Kharissova OV, Jiménez-Pérez VM, Perez BO, et al (2012) Iron-containing nanomaterials: synthesis, properties, and environmental applications. RSC Advances 2: 9325-9358

2. Gao Y, Liu Y, Xu C (2014) Magnetic nanoparticles for biomedical applications: From diagnosis to treatment to regeneration. Engineering in Translational Medicine 567-583.

3. Babes L, Denizot B, Tanguy G, Le Jeune JJ, Jallet P (1999) Synthesis of Iron Oxide Nanoparticles Used as MRI Contrast Agents: A Parametric Study. J Colloid Interface Sci 212: 474-482.

4. Goya GF, Grazu V, Ibarra MR (2008) Magnetic nanoparticles for cancer therapy. Current Nanoscience 4: 1-16.

5. Na HB, Palui G, Rosenberg JT, Ji X, Grant SC, et al. (2012) Multidentate catechol-based polyethylene glycol oligomers provide enhanced stability and biocompatibility to iron oxide nanoparticles. ACS Nano 6: 389-399.

6. Yu MK, Jeong YY, Park J, Park S, Kim JW, et al. (2008) Drug-loaded superparamagnetic iron oxide nanoparticles for combined cancer imaging and therapy in vivo. Angew Chem Int Ed Engl 47: 5362-5365.

7. Daniele MA, Shaughnessy ML, Roeder R, Childress A, Bandera YP, et al. (2013) Magnetic nanoclusters exhibiting protein-activated near-infrared fluorescence. ACS Nano 7: 203-213.

8. Massart R (1981) Preparation of aqueous magnetic liquids in alkaline and acidic media. Magnetics, IEEE Transactions on.

9. Terán JL, Gnecco D, Galindo A, Juárez J, Aguirre-Hernández G, et al. (2001) Crystal structure of (+)-(R)-3-methyl-1-(1'-phenyl-ethyl)-1H-pyridin-2-one. Anal Sci 17: 1247-1248.

10. Chertok B, Moffat BA, David AE, Yu F, Bergemann C, et al. (2008) Iron oxide nanoparticles as a drug delivery vehicle for MRI monitored magnetic targeting of brain tumors. Biomaterials 29: 487-496.

11. Wang $Y$, Xu F, Zhang C, Lei D, Tang Y, et al. (2011) High MR sensitive fluorescent magnetite nanocluster for stem cell tracking in ischemic mouse brain. Nanomedicine: Nanotechnology, Biology and Medicine 7: 1009-1019.

12. Yoon KY, Kotsmar C, Ingram DR, Huh C, Bryant SL, et al. (2011) Stabilization of superparamagnetic iron oxide nanoclusters in concentrated brine with crosslinked polymer shells. Langmuir 27: 10962-10969.

13. Ge J, Hu Y, Biasini M, Beyermann WP, Yin Y (2007) Superparamagnetic magnetite colloidal nanocrystal clusters. Angewandte Chemie International Edition 46: 4342-4345.

14. Bae BC, Na K (2011) Development of polymeric cargo for delivery of photosensitizer in photodynamic therapy. International Journal of Photoenergy 12: 2012.
15. Konan YN, Gurny R, Allemann E (2002) State of the art in the delivery of photosensitizers for photodynamic therapy. J Photochem Photobiol B 66: 89106.

16. Dougherty TJ, Gomer CJ, Henderson BW, Jori G, Kessel D, et al. (1998) Photodynamic therapy. J Natl Cancer Inst 90: 889-905.

17. Calzavara-Pinton PG, Venturini M, Sala R (2005) A comprehensive overview of photodynamic therapy in the treatment of superficial fungal infections of the skin. J Photochem Photobiol B 78: 1-6.

18. Castano AP, Mroz P, Hamblin MR (2006) Photodynamic therapy and antitumour immunity. Nat Rev Cancer 6: 535-545.

19. Thong PS, Ong KW, Goh NS, Kho KW, Manivasager V, et al. (2007) Photodynamic-therapy-activated immune response against distant untreated tumours in recurrent angiosarcoma. Lancet Oncol 8: 950-952.

20. Thong PS, Olivo M, Kho KW, Bhuvaneswari R, Chin WW, et al. (2008) Immune response against angiosarcoma following lower fluence rate clinical photodynamic therapy. J Environ Pathol Toxicol Oncol 27: 35-42.

21. Frangioni JV (2003) In vivo near-infrared fluorescence imaging. Curr Opin Chem Biol 7: 626-634.

22. Yan F, Kopelman R (2003) The embedding of meta-tetra (hydroxyphenyl)chlorin into silica nanoparticle platforms for photodynamic therapy and their singlet oxygen production and $\mathrm{pH}$-dependent optical properties. Photochemistry and photobiology 78: 587-591.

23. Charron G, Stuchinskaya T, Edwards DR, Russell DA, Nann T (2012) Insights into the mechanism of quantum dot-sensitized singlet oxygen production for photodynamic therapy. The Journal of Physical Chemistry C 116: 9334-9342.

24. Prasad PN (2003) Introduction to Biophotonics, Wiley, New York.

25. Meisel P, Kocher T (2005) Photodynamic therapy for periodontal diseases: state of the art. J Photochem Photobiol B 79: 159-170.

26. Hirakawa K, Hirano T, Nishimura Y, Arai T, Nosaka Y (2012) Relaxation Process of Photoexcited meso-Naphthylporphyrins while Interacting with DNA and Singlet Oxygen Generation. J Phys Chem B 116: 3037-3044.

27. Roy I, Ohulchanskyy TY, Pudavar HE, Bergey EJ, Oseroff AR, et al. (2003) Ceramic-based nanoparticles entrapping water-insoluble photosensitizing anticancer drugs: a novel drug-carrier system for photodynamic therapy. J Am Chem Soc 125: 7860-7865.

28. Kumar P, Agnihotri S, Roy I (2016) Synthesis of Dox drug conjugation and citric acid stabilized superparamagnetic iron oxide nanoparticles for drug delivery. Biochem Physiol 5: 194.

29. Sahoo B, Devi KS, Dutta S, Maiti TK, Pramanik P, et al. (2014) Biocompatible mesoporous silica-coated superpara- magnetic manganese ferrite nanoparticles for targeted drug delivery and MR imaging applications. J Colloid Interface Sci. 431: 31-41

30. Kumar P, Anuradha, Roy I (2014) Optically and magnetically doped ormosil nanoparticles for bioimaging: synthesis, characterization, and in vitro studies. RSC Advances 4: 16181-16186.

31. Roy I, Kumar P, Kumar R, Ohulchanskyy TY, Yong KT, et al. (2014) Ormosi nanoparticles as a sustained-release drug delivery vehicle. RSC Advances 4 53498-53504. 